\title{
EL WESTBURN. UN HITO DE LA PRIMERA GUERRA MUNDIAL EN CANARIAS
}

\author{
Alberto García Montes de Oca* \\ José Miguel Rodríguez Illescas** \\ Universidad de La Laguna
}

\section{RESUMEN}

Durante la Primera Guerra Mundial y su gran desarrollo bélico en el entorno europeo y colonial, no se descartaron del conflicto los territorios marítimos neutrales. Éste es el caso de las Islas Canarias, lugar en el que se gestó un novelesco episodio de la Guerra, que tuvo consecuencias a largo plazo y que a día de hoy nos lega un pecio que ha sido objeto de numerosas historias y diversos litigios. En la actualidad el S.S. Westburn, denominado «El Carbonero», yace a las afueras de San Andrés como un hito de la historia bélica en Canarias, dejando en el fondo un pecio categorizado como arqueológico desde el 2016, que ha sido y es objeto de un desmantelamiento y destrucción a conciencia.

Palabras clave: Primera Guerra Mundial, naufragio, arqueología subacuática, legislación sobre patrimonio.

\author{
WESTBURN. A MILESTONE OF \\ WORLD WAR IN CANARY ISLANDS
}

\section{Abstract}

During World War I, and under the whole military progress in Europe and in the colonial territories, marginal lands like islands were not spared from the confrontations. This is the case of the Canary Islands, where an almost comical military event took place, that nevertheless had consequences in the long term and that left us today a valuable shipwreck, that has been the inspiration for many stories and litigation. Today the Westburn, also called "The Coal Steamer," lies in the depths off the San Andrés shore as a milestone of the Canarian Military History. Since 1916, it possesses the status of archaeological shipwreck and has been, and continues to be, a target of dismantlement and conscious destruction.

KeYwords: World War I, shipwreck, subaquatic archaeology, heritage laws. 


\section{CANARIAS DURANTE LA PRIMERA GUERRA MUNDIAL}

Al hablar de la Primera Guerra Mundial nos vienen a la mente las cruentas batallas de Verdún o de Gallipoli, los conflictos armados a gran escala o el desarrollo de la aviación y de la tecnología submarina. Lo alejamos todo del entorno de Canarias, ya que España es Estado neutral, y las Islas, ubicadas en la ultraperiferia de la nación no parecen suponer de cara al exterior un enclave demasiado relevante en el conflicto en lo que se refiere a las grandes potencias de la época. $\mathrm{O}$ al menos eso es lo que vemos en la historiografía general del conflicto. Realmente, se considera a las Islas Canarias como una base en el Atlántico Occidental' ${ }^{1}$. El archipiélago canario ocupa una posición privilegiada con respecto a los demás archipiélagos de la Macaronesia. Su situación frente a la fachada occidental africana y su estratégica posición en las rutas entre Europa, América y África lo hacen único.

Ya desde el descubrimiento y la conquista del continente americano, las Islas desempeñaron un papel fundamental como paso obligado por la navegación, tanto para el avituallamiento de los propios barcos como para el aprovechamiento de los vientos alisios y las corrientes marinas vitales en la navegación de la época, así como para las rutas comerciales y para las militares. Con el paso de los siglos y los correspondientes cambios tecnológicos en la navegación, como el desarrollo del motor a vapor, el Archipiélago incrementó su valor logístico con el avituallamiento de carbón ${ }^{2}$, vital para el funcionamiento de las nuevas técnicas de navegación. Esto, unido al expansionismo de las potencias europeas en su afán de colonizar los territorios africanos, puso a las Islas en el centro de la geoestrategia atlántica.

Es en este marco cuando en agosto de 1914 estalla la Primera Guerra Mundial entre la Triple Alianza, dirigida por el Imperio Alemán, y la Entente, liderada por Gran Bretaña y Francia, entre otros Estados. Conforme se fue desarrollando el conflicto y aunque Espańa se mantuvo neutral durante la guerra, las Islas Canarias se vieron severamente afectadas. Como había ocurrido en otras ocasiones a lo largo de su historia, las Islas poseían fuertes lazos económicos con las mayores potencias europeas ya que suministraban importantes cantidades de productos básicos. El estallido del conflicto supuso un corte en esas relaciones, en su mayor parte, a causa de la preeminencia de las necesidades industriales de guerra de los países beligerantes, haciendo que los mercantes fueran redirigidos a otros puertos y rutas surgidas por y para la guerra.

* Graduado en Historia, Arqueólogo especialista en Arqueología Subacuática. Doctorando en Arte y Humanidades por la ULL.E-mail: albertogmdo@gmail.com.

** Graduado en Historia por la ULL, especialista en Historia Militar Contemporánea por la Universitat Jaume I de Valencia.E-mail: jomirocasa@msn.com.

${ }^{1}$ Documento referente al tema en el Fondo Antiguo del Archivo Municipal de Santa Cruz de Tenerife.

${ }^{2}$ En el caso general, en Canarias se suministraba carbón vegetal. 
A esta nueva situación de guerra en Europa que afectó a Canarias entre 1914 y 1918 debía sumársele la aparición de otro factor nunca visto hasta la fecha: la acción de los submarinos. Si bien con la mayor parte de la flota alemana anclada en puerto debido al bloqueo naval que infligía la Royal Navy sobre el Imperio Alemán, el archipiélago canario se llevó la peor parte de cuanto acaeció al territorio español. El impacto fue muy severo, sobre todo en el comercio y las comunicaciones exteriores debido a la acción de esta nueva tecnología naval. Aunque aún muy primitivos, eran muy efectivos para la interrupción del tráfico mercante aliado y fueron empleados por el Imperio Alemán hasta el final de la contienda.

La acción de los submarinos no fue la única, ya que la actividad de los buques corsarios de ambos bandos fue notable, atacando, apresando y minando las aguas del Archipiélago, lo que agudizó la grave crisis que las Islas soportarían durante cuatro largos años.

La falta de barcos que visitaran los puertos y la paralización del sector exportador produjo la subida del precio de los productos básicos, lo que a su vez acarreó la falta de empleo, así como el aumento y enriquecimiento de los estraperlistas, hecho que propició la emigración a América, especialmente a Cuba.

Otro hecho destacable de este periodo en las Islas es que los escasos buques anclados en puerto son de origen alemán, ya que tras el estallido del conflicto buscaron refugio en las radas canarias. Buques como el Cap Ortegal o el Usambara permanecieron en los puertos canarios, dándose el caso de que uno de estos navíos fue apresado debido a un fuerte temporal que rompió los amarres del barco, sacándolo del puerto. Tras esto, un buque británico armado en corso lo capturó y se lo llevó de las aguas canarias. Otros, como el Walhalla, anclado en el puerto de la Luz se aventuró a salir del puerto y navegó hasta Santa Cruz de Tenerife, siendo todo un alarde de osadía debido a la presencia de buques británicos que patrullaban el canal. Pero sin duda, uno de los hechos más notorios es el hundimiento del vapor S.S. Westburn frente a las costas de San Andrés (Tenerife) en 1916.

Este periodo está plagado de anécdotas, historias y hechos, algunos muy conocidos, muestra de que la Primera Guerra Mundial afectó a Canarias, no solo económica y socialmente, sino también desde el punto de vista militar. Se sucedieron combates y encuentros navales entre el Imperio Británico y el Alemán que propiciaron el hundimiento de un gran número de mercantes civiles y buques militares que a día de hoy permanecen en el fondo del océano como testimonio de esa guerra que desgarró al mundo durante cuatro años y que afectó notablemente al archipiélago canario. Tras éste, no recuperó el volumen de tráfico marítimo anterior a la Primera Guerra Mundial hasta bien entrada la segunda mitad del siglo xx.

\section{LA HISTORIA DEL WESTBURN}

Partiendo de las premisas anteriores, vemos que en el desarrollo del conflicto era frecuente que las redes de aprovisionamiento marítimo atlánticas pasaran por las Islas. En este ámbito, el buque conocido como S.S. Westburn (lámina I), de bandera inglesa, salido en 1893 de los astilleros de la James Westoll Line en 


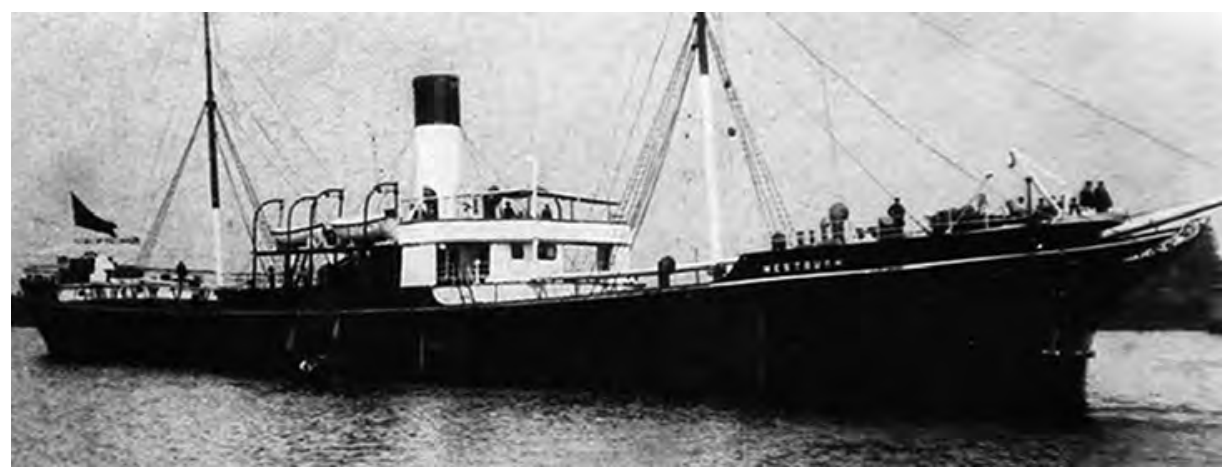

Lámina I. Imagen del S.S. Westburn fondeado en un puerto sin determinar.

Sunderland ${ }^{3}$, navegaba con un cargamento de carbón de Cardiff a Buenos Aires. Se trata de un buque de 3300 toneladas métricas (a partir de ahora TM), casco de hierro con proa tipo violín, de 107 metros de eslora y velocidad punta de siete nudos, dotado de gran bodega y de aparejo de pesca ${ }^{4}$.

El 8 de febrero de 1916, el buque Möwe, armado en corso al servicio del Imperio Alemán, lo capturó en alta mar a unas 500 millas de Pernambuco, Brasil. Transfirieron a los presos de otros cinco buques atacados por el corso, haciendo un total de 199 reos, y ambos barcos, el Möwe y el Westburn, se dirigieron rumbo nornoreste, hacia la Macaronesia, contando éste último con un oficial y seis tripulantes del Möwe, que velaban por los intereses alemanes en el carguero. Según testimonios presenciales, se mantuvo a raya a los reclusos con cargas explosivas escondidas en el buque, lo que facilitó su sumisión ${ }^{5}$.

El Westburn es avistado desde el día 22 de febrero, cuando llega al puerto de Santa Cruz de Tenerife (lámina II) desde la costa suroriental de la Isla ${ }^{6}$ sin bandera en su pabellón, eludiendo al crucero inglés que vigilaba la zona, el Sutlej. Al entrar en el puerto, enarbola la bandera de guerra alemana, determinando que es una captura de guerra, pero atendiendo al convenio de La Haya de 1907, no podía ser capturado en puerto neutral'.

3 Junto a Newcastle, en la costa nororiental de Inglaterra.

${ }^{4}$ Dominio web de Sunderland, p. 29: armadores, p. 23.

${ }^{5}$ Referentes a este acontecimiento citamos la primera plana de al menos tres periódicos de la época: el primero, El Progreso, con fecha de 24 de febrero de 1916; el segundo, La Opinión, en su tirada del 23 de febrero de ese año -noticiarios que por su cercanía al evento consideramos como fuentes más fidedignas-, y representando la relevancia del acontecimiento, The New York Times, en su tirada del día 28 del citado mes.

${ }^{6}$ Unas millas antes, el Möwe tomó rumbo norte antes de adentrarse en el Archipiélago.

7 Convención relativa a los Derechos y Deberes de las Potencias Neutrales en la Guerra Marítima, La Haya, el 18 de octubre de 1907. 


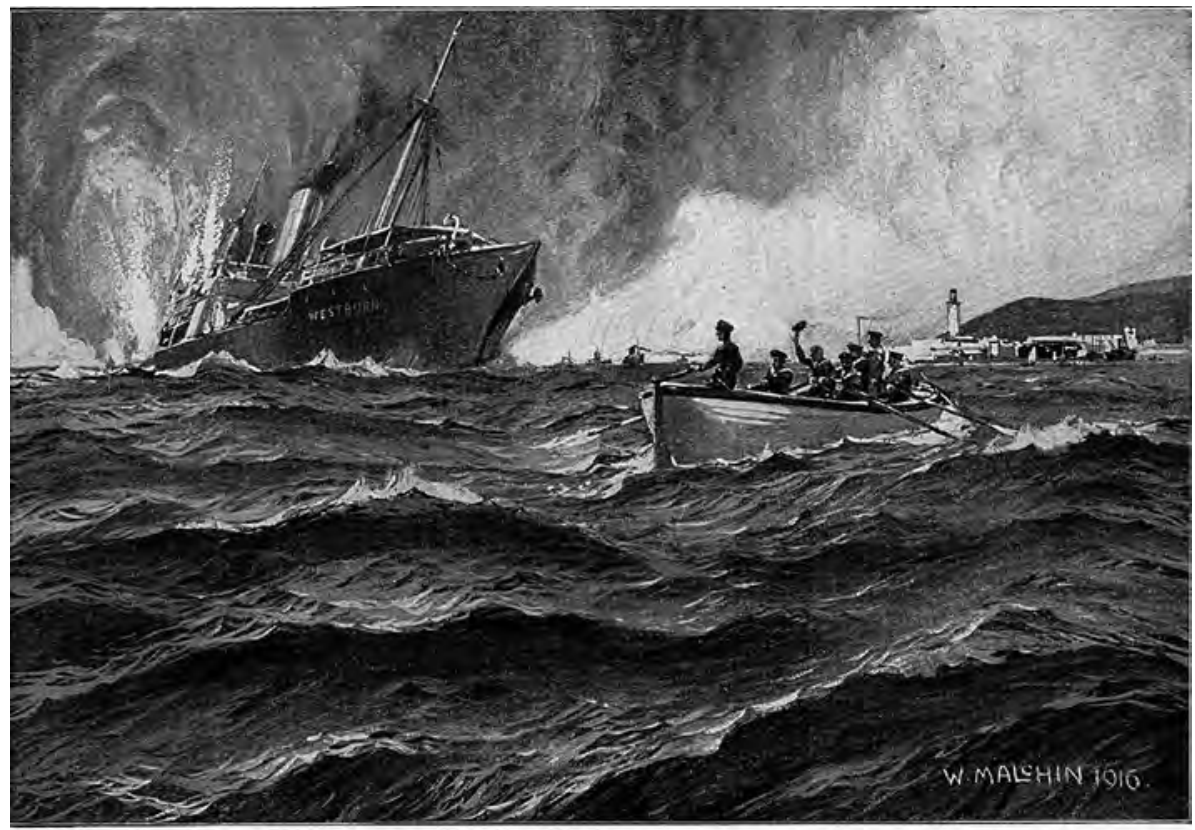

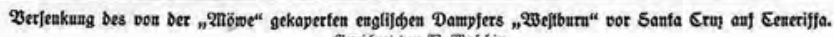

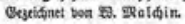

Lámina II. Dibujo de W. Malchin, publicado en una revista austríaca de 1916. Colección de Agustín Miranda Armas.

Los prisioneros desembarcan y en los días posteriores se repatrian a sus Estados de origen. En cuanto al barco y su dotación alemana, habiendo eludido al buque Essex y luego al Sutlej, no pasó sino unas horas en el puerto de Santa Cruz, sin siquiera visitar ni dar cuenta al cónsul de Alemania en la Isla de su paso por ella. Lo encaminaron rumbo norte hasta hacer través con la playa de Las Gaviotas y parando sus máquinas, detonaron cargas explosivas justo después de salir del barco ${ }^{8}$. El S.S. Westburn es hundido en el lugar, donde aún permanece, a una profundidad de entre 32 y 36 metros.

Los alemanes, ahora prisioneros (lámina III), fueron conducidos a Capitanía, donde se solicitó un traductor para su declaración. Los prisioneros eran: el capitán Reinhold Badewitz ${ }^{9}$ la tripulación de seis compuesta por el primer maquinista Franz Schreck ${ }^{10}$, el contramaestre Ludwig Wahls, los cabos de mar de primera Dietrich

${ }^{8}$ Queremos destacar que el hundimiento, a las 16:00 horas, es avistado por un tumulto de gente, muchos de ellos germanófilos, que estaban al tanto de lo que iba a ocurrir.

${ }^{9}$ Llamado en diversos informes Baderwitz.

${ }^{10}$ Realmente cabo de mar de primera, pero con dicha función en diversos barcos en los que navegó. 


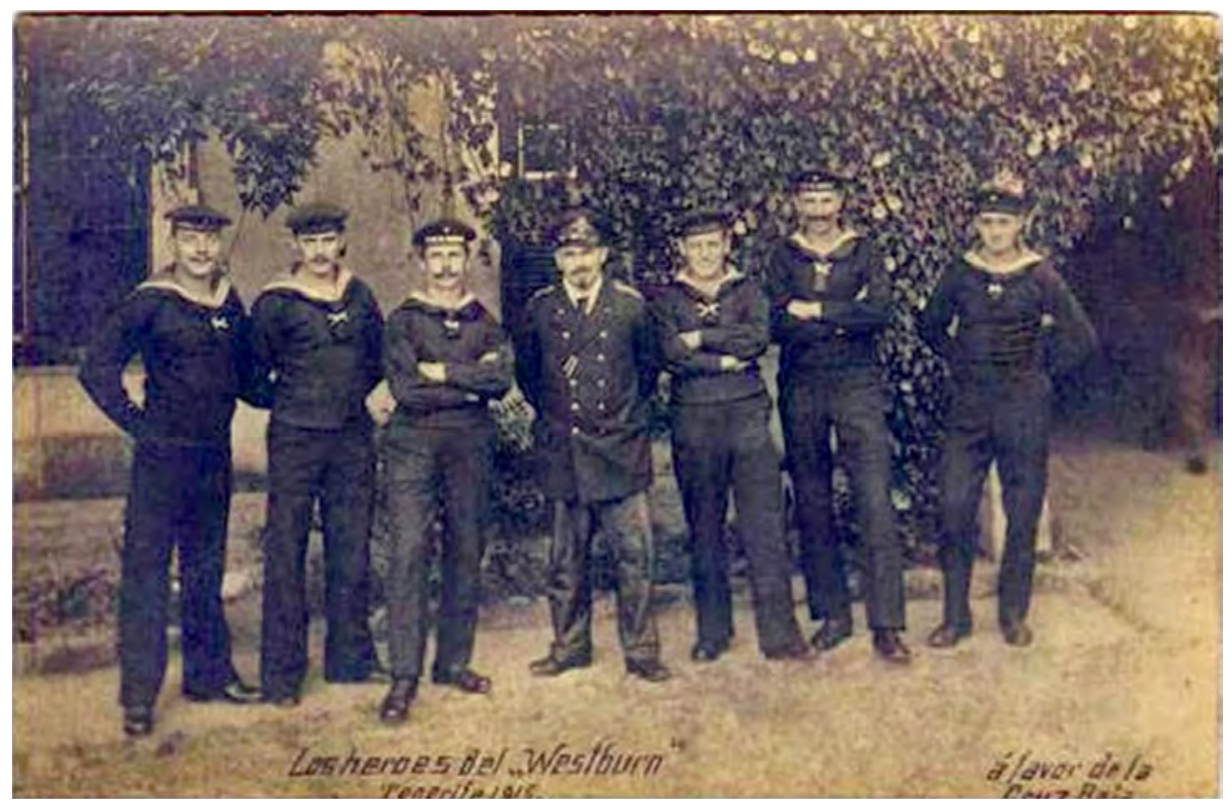

Lámina III. Fotografía titulada "Los Héroes del Westburn», que muestra a los siete prisioneros alemanes capturados tras el hundimiento del Westburn.

Bueltmann y Franz Henschen, y los cabos de mar de segunda Heinrich Juelich y Georg Gornig. Fueron llevados al destacamento de Artillería de Almeida, para ser suministrados y atendidos por el coronel de Artillería de la plaza. Se permite bajo permiso expreso al secretario del cónsul Imperial Verner Freiherr, visitar a los cautivos en Almeida para entrevistarlos.

A partir de ahí, más que prisioneros parecen invitados. Los llevan a pasear por La Orotava y de excursión a Las Mercedes. Aunque se quiere juntar a todos los prisioneros alemanes de las Islas en Las Palmas, se acuerda no hacerlo ya que el canal entre las dos islas es frecuentemente patrullado por ingleses y se quiere dar la oportunidad a los alemanes de "gozar un mejor trato" en territorio neutral sin ser apresados ${ }^{11}$. Prometen no escapar bajo palabra de honor, y que, de cambiar de parecer, darían cuenta de ello retirándola a través del cónsul alemán. Con ello, los privilegios a los prisioneros aumentan y se les permite salir del cuartel durante las

11 Aunque con ello se respete la Convención relativa a los Derechos y Deberes de las Potencias Neutrales en la Guerra Marítima mencionado anteriormente, puede que aquí veamos un cierto posicionamiento de las instituciones militares a favor de los alemanes, y se atisba un cierto recelo hacia los ingleses, tan en conflicto con España durante toda la Edad Moderna y fechas posteriores. Con este ejemplo puede hacerse patente la división de opiniones entre los españoles: anglófilos y germanófilos. 
horas diurnas, especialmente al oficial Badewitz. Reciben el capitán Badewitz y el primer maquinista Schreck consideración de oficiales y son tratados como tales. Pero los derechos afines a esta designación se deniegan a Schreck por ser cabo.

Los privilegios al amparo del cónsul aumentan y se permite a los prisioneros asistir a las fiestas de mayo, comer en casas de familias alemanas, ir al teatro y de excursión, siempre que vuelvan a pernoctar al cuartel. Pero el 4 de julio de ese ańo, llegó la situación en la que Badewitz y Freiherr retiraron su palabra de honor ${ }^{12}$ de no escapar y pasaron al cargo de las autoridades militares. Solo era necesario conceder a los prisioneros, en especial al capitán Badewitz, determinados privilegios para que sintiesen que ya no tenían más retos con los españoles y especialmente con los militares. Tras varias quejas por su nuevo trato como prisionero, fue finalmente enviado a Las Palmas junto a su tripulación en el crucero español Princesa de Asturias. En Gran Canaria fueron recluidos. El 20 de agosto, en un descuido del cuerpo de guardia, se fugó con la ropa que le dejaron unos visitantes en su celda. El resto de prisioneros fueron repatriados en los años posteriores.

Desde este punto, saltamos a los inicios de la década de 1940, cuando volvemos a tener constancia del barco. Para entrar en detalles, la Ley de Enjuiciamiento Militar de 1886 (González Padilla, 2002: 155-166) era la vigente en ese entonces en referencia al hallazgo y salvamento de embarcaciones y cosas hundidas, centro del litigio que podemos analizar hasta la actualidad.

Entrando en detalles ${ }^{13}$, al renacer la constancia de que en el fondo de nuestras aguas existía un carguero repleto de carbón inexpugnado desde hacía casi treinta años, la Agrupación de Pescadores de Santa Cruz, en vinculación con la Casa Hamilton \& Cía., acordaron extraerlo. La compañía pondría los buzos, sería depósito del material extraído y se encargaría de la gestión comercial, mientras que la Agrupación de Pescadores, representada por Industrias Marítimas de Tenerife, Pesquerías y Salazones, Eduardo Coll Díaz, Salvador Vázquez y Paulino Freire, aportaría el resto de personal y medios para la contienda. Sin contar con la supervisión económica estatal, pero dando siempre cuentas a la Comandancia de Marina de Santa Cruz de Tenerife para que estuviese al tanto de los trabajos, la Compañía Hamilton encargó a Paulino Freire la tasación del carbón. El precio estimado para cada una de las casi 5000 TM de carbón fue de 63,33 pesetas, al igual que el precio del carbón de León y Palencia en 1942.

Las primeras extracciones se sucedieron sin problemas y el carbón era depositado en los almacenes de la Compañía Hamilton. Tras ello, se comienza a garantizar el aprovechamiento del mineral. En una carta del Juzgado de Instrucción de Marina de Tenerife al Jefe del Departamento Marítimo de Cádiz, acompañada de una notificación del capitán general de Canarias, se avisa que debido a la escasez

12 En referencia al artículo 24 de la Convención relativa a los Derechos y a los Deberes de las Potencias Neutrales en la Guerra Marítima, tan en boga en este escrito.

${ }^{13}$ Esta información está extraída de los informes del expediente instruido de extracción de carbón del vapor Westburn del Departamento Marítimo de Cádiz y la Comandancia Naval de Santa Cruz de Tenerife, depositados en el Archivo Intermedio de Almeida. 
de combustible existente en Canarias, el carbón no sería enviado a la Península, sino que sería para uso de primera necesidad, en especial para abastecer a los pesqueros de la Isla y que pudieran salir a faenar.

Los problemas comienzan al notificarse en febrero de 1943 a través de la Compañía Hamilton, representante en Canarias de la Salvage Association, que reclama su derecho sobre la carga del buque. El fiscal del Departamento Marítimo manda que se siga la Ley de Enjuiciamiento Militar y que se presenten los documentos que avalen su derecho sobre la propiedad del carbón, gestionando los militares el derecho a extracciones de un cargamento inicialmente privado, que se acoge al amparo español a través de su legislación.

Seguidamente, aparece Pedro del Toro Santana, que alega que el capitán general y el almirante Jefe del Estado Mayor de la Armada le habían concedido la potestad de la extracción, al estar en conocimiento del material hundido desde hacía ya varios lustros y tener intención de explotarlo. En marzo de ese año, se le concede el legítimo derecho, pero al poco, la potestad pasa a José María de la Concha al no emprender el primer concesionario ningún trabajo de extracción. Al mismo tiempo, la Salvage Association no puede, por la guerra, avalar su título de propiedad sobre el mineral.

En un tira y afloja por establecer el valor del carbón, pudiendo sacar beneficio los encargados de la extracción bajo el mando de José María de la Concha, se estimó el precio por TM en 63,33 pesetas tal y como vimos antes, para venderlas a mayor precio después. Se llega a la conclusión que el carbón Cardiff que tiene el barco es de la mejor calidad y que el precio dobla y supera la cantidad estimada con creces, con lo que el juez instructor de la Comandancia Militar declara que se han defraudado al Tesoro los impuestos de 240000 pesetas en las que estaban valoradas las primeras $600 \mathrm{TM}$ de carbón extraídas, siendo por tanto su valor real de 400 pesetas y no 63. Con ello, se aludió al tráfico fraudulento que evadía las aportaciones al Tesoro. En ese informe, de igual forma, se denuncia el desguace chatarrero de la estructura del barco, denominando la dirección del salvamento de «descuidada, poco perita y ciega de codicia».

El informe no tiene desperdicio y denota el enfado del juez instructor, lo que no es para menos. Tras quitar todas las tuberías, las piezas de valor, la campana de bronce ${ }^{14}$ y la maquinilla de cubierta, se pretendía dinamitar la estructura del barco para sacar todo el carbón que quedaba, diseminándolo, haciéndolo inviable y llenando el interior del pecio de arena. Las quejas no fueron pocas y el director de la extracción del carbón, José María de la Concha Palacios, fue acusado, sin mucho desatino, de especulación y fraude al Tesoro, pero en defensa de su honor, declaró que la entidad en la que se depositaba el carbón, la Casa Hamilton, era la encargada de efectuar ese pago. Sea como fuera, la documentación alude a las posteriores ventas de carbón según la primera tasación: 64 pesetas.

${ }^{14}$ Cedida y colgada en la ermita de San Andrés, donde se puede ver hoy en día. 
En la línea de acontecimientos, la cordura no duraría mucho, ya que el comandante de Marina de Santa Cruz de Tenerife, el 11 de junio de 1943, autorizó a Raimundo Delgado Santana a colocar cargas de dinamita distribuidas por el buque para sacar el contenido restante, pese a haberse estipulado que se mantendría la integridad del mismo. El 8 de marzo de 1944, Delgado y su socio De la Concha, renuncian expresamente a su derecho a seguir con las extracciones. Seguidamente, en mayo de ese año, a través del capitán general del Mando Económico de Canarias, se designa concesionario a Alfonso Pérez Delgado, bajo las mismas condiciones contractuales.

Aprovechando el nuevo giro, el fiscal del Departamento Marítimo de Cádiz resuelve que no se trata de un hallazgo, como se había planteado anteriormente, causa a través de la cual se pudo gestionar la extracción a través de la Ley de Enjuiciamiento Militar, sino que, al conocerse siempre su paradero, solo se trataba de una concesión para la extracción de combustible de un pecio ya conocido. Esta sentencia abrió un debate jurisdiccional, del que podemos ver referentes en Arqueología Subacuática y problemática de potestades en la actualidad ${ }^{15}$.

Se concede de esta manera al capitán general, jefe del Mando Económico de Canarias, la autoridad para designar a quien él considere oportuno para los trabajos, ya que finalmente se determina lo mencionado anteriormente, que no se trata de un hallazgo y no se acoge por tanto a los artículos 42 y 43 de la Ley de Enjuiciamiento Militar. La decisión se toma en el seno de una región que no disponía de carbón para sustentar sus actividades, ni siquiera las de los pesqueros para suministrar alimento. Tras diversas reclamaciones, la Salvage Association a través de la Compañía Hamilton, acepta la resolución última en febrero de 1946. El caso se cierra definitivamente en 1955 .

\section{LA ACTUALIDAD DEL WESTBURN}

Hoy en día, tras haber pasado más de 100 años desde su hundimiento, y siguiendo las premisas de la Convención de la UNESCO de 2001 para la Salvaguarda del Patrimonio Arqueológico Sumergido, el pecio se considera patrimonio arqueológico subacuático.

Cierto es, que nunca estuvo olvidado y se han hecho a lo largo de los años extracciones y actividades en él, pero es susceptible de ser estudiado bajo metodología arqueológica. Como patrimonio de tales características, se acoge a la protección española al estar hundido en aguas territoriales nacionales. Protección a través de la labor de la Armada, que se comprometió con su participación en el Plan Nacional de Protección del Patrimonio Arqueológico Subacuático de 2010, reeditado en 2015; y a

15 Dicho informe es el expediente del Ministerio de Marina para determinar la propiedad del vapor inglés Westburn, hundido en las aguas de Tenerife en el mes de febrero de mil novecientos dieciséis. 
través de la Ley de Navegación Marítima de 2014. Según las características del barco: pecio arqueológico, de bandera extranjera, sin la característica de buque de Estado y conocida su ubicación, las competencias de gestión sobre el mismo las tiene en la actualidad el Gobierno de Canarias, a través de la Dirección General de Patrimonio Cultural. Respecto a las actuaciones sobre su patrimonio biológico asociado y la extracción de material, éstas las regula el Ministerio de Medio Ambiente ${ }^{16}$, a través de la Dirección Generale Costas, siendo por tanto encargada de su protección la Armada española, que habrá de ser notificada ante cualquier intervención.

Hoy, «El Carbonero» yace frente a las costas de San Andrés y es visitado asiduamente por buceadores deportivos, que en innumerables ocasiones se llevan «algún recuerdo». Esta actividad no está vigilada y se considera irrisorio un fragmento de carbón que alguien se pueda llevar. Dentro del amasijo de hierros que es hoy el Westburn, no hay conciencia de perdurabilidad. Extraer un trocito de carbón no supondría un problema ético si éste fuese ilimitado. Pese a poder cogerse antes de 2016 sin ninguna connotación legal, los 100 ańos y las nuevas características atribuidas al pecio hacen que esto sea ilegal. De esta manera, hemos de difundir la idea de cuidar nuestro patrimonio, sea natural, arqueológico o cultural, educando e integrando en la ciudadanía la conciencia de cuidar lo que es de todos.

RECIBIDO: 02-02-2018, ACEPTADO: 10-04-2018

${ }^{16}$ Plan Nacional de Protección del Patrimonio Arqueológico Subacuático, 2015: 11. 


\section{DOCUMENTACIÓN}

\section{Artí́culos}

GonzÁlez Padilla, E. (2003): «La Justicia Militar en el Primer Franquismo», en Gutiérrez Navas y Rivera Menéndez, J.: Sociedad y politica durante el régimen de Franco. Actas de las Jornadas celebradas en la UNED durante los dias 8 al 12 de abril de 2002 (155-166), Almería, Instituto de Estudios Almerienses.

\section{Prensa}

a) Anónimo (1916, 23 de febrero) «Suceso Emocionante». La Opinión, 7068.

b) Anónimo (1916, 24 de febrero) «La Voladura del Westburn». El Progreso, 3194.

c) Anónimo (1916, 28 de febrero) «How Westburn’s Crew Missed Crusoe’s Fate». The New York Times.

\section{Documentos inéditos De ARChivo}

Documentación referente al Westburn, años 1916 y 1942-1945.

\section{Fuentes electrónicas}

a) Dominio web de Sunderland, p. 29: armadores, p. 23: http://www.searlecanada.org/sunderland/ sunderland079.html\#shortbrothers99626.

b) Información sobre pecios ingleses hundidos y su ubicación: http://www.naval-history.net/WW1NavyBritishShipsLocations10_AttackedMNDate1916.htm.

c) Cuaderno de a bordo del Sutlej, febrero 1916: http://www.naval- history.net/OWShips-WW1- 05HMS_Sutlej.htm.

\section{Publicaciones oficiales}

a) Bélgica, La Haya (1907): Convención relativa a los Derechos y a los Deberes de las Potencias Neutrales en la Guerra Marítima.

b) Ley 16/1985, de 25 de junio, de Patrimonio Histórico Español, Boletín Oficial del Estado, Madrid, 29 de junio, núm. 155, 20342-20352.

c) Ley 22/1988, de 28 de julio, de Costas, Boletín Oficial del Estado, Madrid, 29 de julio, núm. 181, 23386-23401.

d) Ley 4/1999, de 15 de marzo, de Patrimonio Histórico de Canarias, Boletín Oficial de Canarias, Canarias, 9 de abril, núm. 85, 13278-13299.

e) Decreto 262/2003, de 23 de septiembre, por el que se aprueba el reglamento sobre intervenciones arqueológicas en la Comunidad Autónoma de Canarias, Boletín Oficial de Canarias, Canarias, 6 de octubre, núm. 194, 15942-15948.

f) Decreto 111/2004, de 29 de julio, por el que se aprueba el Reglamento sobre el Procedimiento de Declaración y Régimen Jurídico de los Bienes de Interés Cultural, Boletín Oficial de Canarias, Canarias, 13 de agosto, núm. 157, 12813- 12818.

g) Ley 14/2014, de 24 de julio, de Navegación Marítima, Boletín Oficial del Estado, Madrid, 25 de julio, núm. 180, 59193-59311.

h) Plan Nacional de Protección del Patrimonio Arqueológico Subacuático (2010), Misisterio de Educación, Cultura y Deportes, 2015. 
